\title{
Applications of Femtochemistry to Proteomic and Metabolomic Analysis
}

\author{
Xin Zhu, ${ }^{\dagger}$ Christine L. Kalcic, ${ }^{\dagger}$ Nelson Winkler, ${ }^{\dagger}$ Vadim V. Lozovoy, ${ }^{\dagger}$ and Marcos Dantus ${ }^{*, \dagger,}$ \\ Department of Chemistry, Departments of Physics and Astronomy, Michigan State University, \\ East Lansing, Michigan 48824
}

Received: January 4, 2010; Revised Manuscript Received: August 6, 2010

\begin{abstract}
Femtosecond laser pulses have been widely used as a tool to study molecular ionization and fragmentation. This article bridges the application of femtosecond laser technology in early research focused on small isolated molecules with that in modern biological mass spectrometry for proteomics and metabolomic analysis on large $(140+$ atoms) biomolecules. The single-shot interaction of a femtosecond laser with neutral paranitrotoluene $(p N T)$ is investigated with time-of-flight mass spectrometry and compared with the ultrafast photodissociation of protonated $p$ NT in an ion trap mass spectrometer accumulated over $\sim 1000$ pulses. The ion trap experiment is then extended to longer biomolecules. As demonstrated in the examples of vasopressin and tomatine, this novel ion activation method provides greater sequence coverage and nonstatistical fragmentation, leading to valuable information complementary to conventional methods for structural analysis.
\end{abstract}

\section{A. Introduction}

The use of femtosecond lasers to provide a dynamic understanding of chemistry, Femtochemistry, has traditionally been part of an academic pursuit toward understanding the most fundamental steps of chemistry. Early studies on the dynamics of chemical reactions, including the time it takes to make or break a chemical bond, were recognized by the 1999 Nobel Prize in Chemistry. ${ }^{1}$ This article describes how a fundamental study in femtosecond laser control in ionization and fragmentation of isolated molecules has been transformed into a novel ion activation method that promises to provide valuable information for proteomic and metabolomic analysis. ${ }^{2}$ The emerging fields of proteomics and metabolomics depend on the structural elucidation of proteins and metabolites. Mass spectrometry (MS) has emerged as the instrument of choice, given that analyses require only a few microliters of analyte in micromolar or nanomolar concentrations. The ability to induce molecular fragmentation is of interest for proteomic and metabolomic analysis because the resulting fragment ions detected provide clues that are used to elucidate molecular structure, protein sequencing being a prime example.

Mass spectrometry instruments designed for molecular structure analysis employ one or more methods for inducing ion fragmentation, or as is known in MS, ion activation. The most common activation method is collision-induced dissociation (CID). During CID, the ions of interest collide with an inert bath gas such as helium, and the energy deposited by the multiple collisions leads to fragmentation. In the case of proteins, CID leads to cleavage of some peptide bonds, and this provides valuable information required for protein sequencing. The thermal nature of CID leads to a statistical distribution of energy and the preferential cleavage of weak chemical bonds over strong ones. This observation has led to the search for methods that are able to achieve nonstatistical bond cleavage. Among the most salient advances in this regard is UV laser-induced photodissociation, which is very effective in the presence of

\footnotetext{
* Corresponding author. E-mail: dantus@msu.edu.

Department of Chemistry.

* Departments of Physics and Astronomy.
}

chromophores such as aromatic amino acids. ${ }^{3,4}$ Although UV fragmentation of peptides is usually achieved using nanosecond laser pulses, some pump-probe experiments have recently been carried out on small protonated chromophores using femtosecond 266 and $405 \mathrm{~nm}$ pump lasers. ${ }^{5-7}$ Another important development is the introduction of electrons which, upon collision with the precursor ion, are able to deposit large amounts of energy, which may lead to nonstatistical bond cleavage. These methods include electron capture dissociation ${ }^{8}$ and electron transfer dissociation. ${ }^{9}$ Both of these methods provide valuable information that is complementary to CID. Their only drawback is that electron capture leads to a reduction of positive charge; therefore, these methods are not compatible with low-charge peptides, such as those produced by one of the most popular sample introduction methods, matrix-assisted laser desorption ionization.

The use of femtosecond laser pulses opens a new approach to ion activation. The interaction of an ultrafast laser pulse and an ion is very different from that of a nanosecond laser pulse, especially when the photon energy is much smaller than the ionization energy. In general, ionization of a neutral molecule requires 7-9 eV of energy, while further ionization of trapped protonated peptide ions requires $10.5-11.5 \mathrm{eV}$ of energy. ${ }^{10}$ This energy can be provided through a nonlinear optical interaction between a long wavelength laser (with photon energy much smaller than that required for ionization) and the molecule. The ionization may proceed through a multiphoton process or through field-induced over-the-barrier ionization. The difference between these two processes depends on the intensity and wavelength of the laser. When the wavelength of the laser is short, ionization takes place during a number of optical cycles; however, when the wavelength of the laser is long and the intensity of the field is high, ionization may take place during a single optical cycle. ${ }^{11}$

Intense femtosecond lasers in the near-IR regime are ideal sources for the activation of molecules and ions through fieldinduced ionization as described above. For these sources, assuming the laser has a photon energy of $1.5 \mathrm{eV}$ and the ionization energy is $9 \mathrm{eV}$, an intensity of $10^{14} \mathrm{~W} / \mathrm{cm}^{2}$ is sufficient to be in the above-barrier ionization regime. At this level of 
BOND SELECTIVE OPTIMIZATION IDENTIFICATION

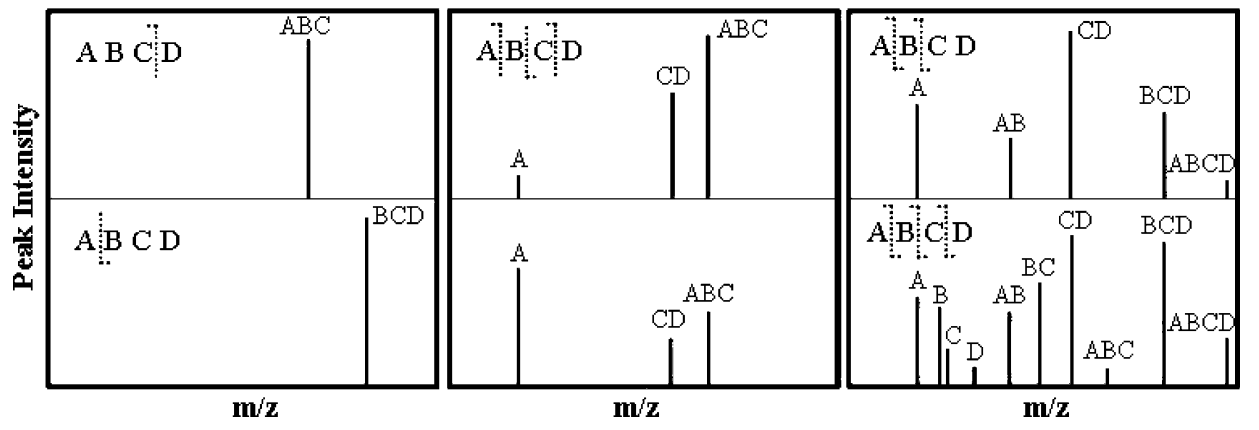

Figure 1. Cartoon illustrating the different goals in molecular fragmentation.

power density, most of the molecules are ionized/activated. The experimental value for ionization saturation for most organic molecules is $1-2 \times 10^{14} \mathrm{~W} / \mathrm{cm}^{2}{ }^{2}{ }^{12,13}$ An observation that caused real interest was that shaping the femtosecond laser pulses results in very different product ion distributions, and this led to several studies in laser control of fragmentation. ${ }^{14}$ Recently, our group reviewed these efforts and systematically evaluated which parameters of the field were responsible for the different fragmentation pathways. ${ }^{15}$ Our conclusion was that pulse duration was the most important parameter provided that the resulting ions were able to undergo further fragmentation by absorbing additional photons of the incident field. We tested our observation on 16 different molecules, including some that had been studied by other groups, and in all cases, pulse duration was a good predictor of fragmentation pattern. Following that study, our attention turned to the activation of trapped ions as used in mass spectrometry for the study of biomolecules.

To place the different goals that may be pursued when using a femtosecond laser to fragment a molecule into perspective, we make use of the cartoons depicted in Figure 1. In the first panel (left), we illustrate a simplified interpretation of the goal known as bond-selective laser control of chemical reactions, by envisioning the resulting MS data for two different laser pulses that are optimized to cleave two different chemical bonds. With the exception of molecules such as HOD, for which intramolecular vibration relaxation occurs in a very slow time scale, there are very few examples in the literature in which bond-selective laser-induced cleavage has been observed.

The second panel (middle) illustrates the type of controllability that is typically observed when near-IR femtosecond laser pulses are shaped. When the laser pulse is shortest, one usually observes larger fragment ions (top), and when the laser pulse is shaped, one observes that smaller fragments have greater relative amplitude (bottom). Notice that in these types of experiments, it is unusual to observe the appearance/disappearance of molecular fragmentation pathways. In other words, unlike the panel on the left, pulse shaping typically leads only to changes in amplitude. The recent work on a small neutral peptide analog by Laarmann et al. is a relevant illustration of these efforts. ${ }^{16}$

In the last panel of Figure 1 (right), we illustrate the type of ion-fragment data that would be required to deduce the bond sequence in the prototypical protein. In the top panel, we envision a method that provides limited bond fragmentation, whereas the bottom panel shows the ideal case in which the fragments produced provide $100 \%$ structural coverage or sequence coverage (in the case of a peptide) for this molecular species. Cleavages that occur along the backbone of the peptide are denoted using a standard nomenclature. ${ }^{17}$ These labels convey both the identity of the broken bond and the number of amino acid residues remaining in the fragment ion, and which end of the molecule has retained the charge, allowing the fragment to be detected. For example, $a b_{7}$ ion is generated through the cleavage of a peptide $(\mathrm{C}-\mathrm{N})$ bond that leaves the seven amino acids at the $\mathrm{N}$-terminal end of the peptide linked together and protonated, whereas a $\mathrm{y}_{3}$ ion is generated through the cleavage of a similar peptide bond, yet this time the three amino acids at the $\mathrm{C}$-terminal end of the molecule retain the extra charge and are detected. With a series of these ions, the mass difference between neighboring ions can be used to identify the consecutive amino acids, revealing the peptide sequence. Each of the amino acids has a unique molecular weight, with the exception of the structural isomers, leucine and isoleucine. Fragments involving the side chain groups must be employed to distinguish between these two amino acids. Although simple MS/MS spectra can be analyzed in this manner, computer software is available for elucidating protein sequences from more complex spectra. From this standpoint, the greater the quantity of product ions in any given MS/MS spectrum, the greater our ability to assign a structural sequence with good statistical confidence.

The activation of trapped ions can be achieved by using ultrafast long-wavelength pulses through field-induced over-thebarrier ionization, as discussed above. The ion of charge $n$ is ionized to produce a radical ion of charge $n+1$. The newly created ion can also acquire additional energy, which leads to fragmentation. The processes that become available with ultrafast lasers with long wavelengths can be used (i) for increasing the charge of trapped ions and (ii) to fragment both strong and weak bonds in trapped ions in a nonstatistical fashion. This latter case may be important for the analysis of posttranslational modifications (PTM) of proteins. Living organisms use PTMs for optimizing the structure and function of proteins. PTMs include the addition of small molecules such as acylation, alkylation, amidation, glycosylation, lipoylation, phosphorylation; the addition of a peptide, as in ubiquitination; changing the nature of amino acids, such as in eliminylation; changing the structure, as in disulfide bridges; or racemization. Certain PTMs have been linked to specific diseases, to aging, and as markers for stress; therefore, their identification is of pharmaceutical and medical importance.

This article presents results on the interaction of near-IR femtosecond laser pulses with isolated neutral and ionic molecular species ranging from small organics (15 atoms) to large biomolecules ( $140+$ atoms). The experimental section describes the instruments and modifications required to interface an ultrafast laser with an ion-trap mass spectrometer to conduct femtosecond laser-induced ionization/dissociation (fs-LID) experiments. Results are presented for para-nitrotoluene $(p \mathrm{NT})$, to explore the different fragmentation pathways between its 


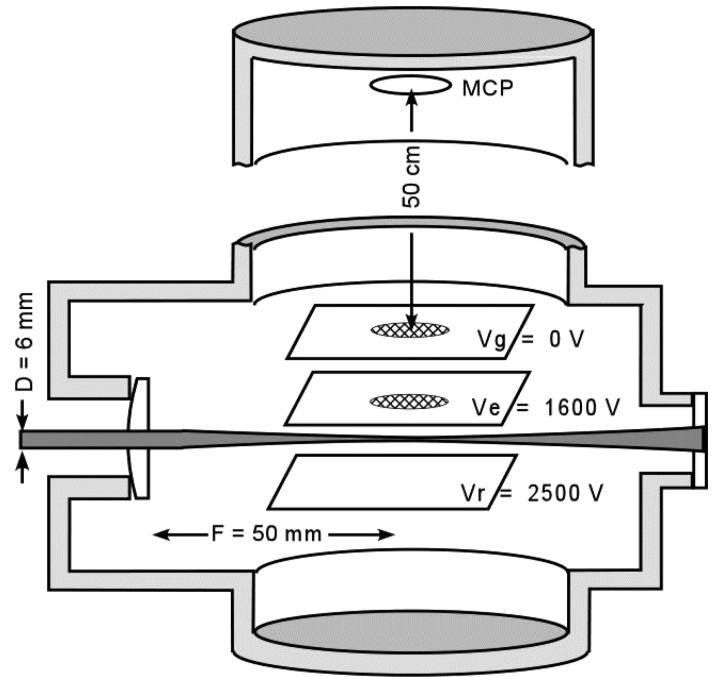

Figure 2. Schemetic of the time-of-flight mass spectrometer. The laser beam is introduced into the chamber through a lens. Ions, generated between the repeller and extractor at high voltage, are detected by the dual microchannel plate detector after a $0.5-\mathrm{m}$ field free flying region.

neutral and protonated forms. Finally, we present results obtained for a protein and a large metabolite to highlight the promise of this approach to solve important proteomic and metabolomic problems of current interest in biological mass spectrometry.

\section{B. Experimental Section}

Experiments were carried out using a regeneratively amplified $\mathrm{Ti}: \mathrm{Al}_{2} \mathrm{O}_{3}$ laser seeded with a broad-band $\mathrm{Ti}: \mathrm{Al}_{2} \mathrm{O}_{3}$ oscillator. The output was centered at $800 \mathrm{~nm}$ with the maximum pulse energy of $\sim 800 \mu \mathrm{J}$. The bandwidth was $\sim 28 \mathrm{~nm}$ (fwhm), resulting in $\sim 35 \mathrm{fs}$ (fwhm) transform-limited (TL) pulses. A MIIPS-enabled pulse shaper ${ }^{18}$ was placed before the amplifier to eliminate phase distortions from the laser and any optics in the setup and to deliver arbitrary phases precisely at the target. Experimental samples of para-nitrotoluene (Aldrich >99\%) and vasopressin (Aldrich, >97\%) were used without further purification, and tomatine was isolated from a tomato plant in-house.

fs-TOF. The fs-TOF experiments were carried out using a time-of-flight mass spectrometer (TOFMS) with a $0.5 \mathrm{~m}$ fieldfree drift region (Figure 2). Sample molecules were effused through an inlet valve into the chamber, where the pressure was maintained with a three-stage differentially pumped system at $10^{-7}$ Torr with operational flexibility up to $10^{-5}$ Torr during experiments. Pulses with a $1 \mathrm{kHz}$ repetition rate were focused by a $50 \mathrm{~mm}$ lens into the chamber to cause ionization and fragmentation of the molecules. The energy per pulse was attenuated to $100 \mu \mathrm{J}$ to reach a corresponding peak power density of $4.0 \times 10^{15} \mathrm{~W} / \mathrm{cm}^{2}$.

fs-LID. The amplified Ti:sapphire laser was interfaced with a modified Thermo Scientific LCQ DECA XP Plus ion trap mass spectrometer (Figure 3). The laser delivered transform limited pulses (35 fs duration, $200 \mu \mathrm{J} /$ pulse, $1.6 \times 10^{14} \mathrm{~W} / \mathrm{cm}^{2}$ calculated peak power density) to the center of the ion trap at a repetition rate of $10 \mathrm{kHz}$. Solutions of $p \mathrm{NT}$, vasopressin, and tomatine were prepared at $1 \mathrm{mg} / \mathrm{mL}$ concentration in 50:50 (v/v) $\mathrm{MeOH} / \mathrm{H}_{2} \mathrm{O}$ spiked with $1 \%$ acetic acid and were introduced to the mass spectrometer by electrospray ionization. Precursor ion populations were isolated and then subjected to MS/MS by fs-LID or CID. A computer-controlled shutter was used to limit irradiation to fit within the ion activation interval of the standard MS/MS experiment. Irradiation times of 100-300 ms were used to generate fs-LID spectra with good signal-to-noise ratios, corresponding to the excitation and dissociation of the trapped ion packet after interaction with 1000-3000 laser pulses.

\section{Results}

Mass spectra of $p \mathrm{NT}$ obtained by electron ionization (EI), fs-TOF, CID, and fs-LID are shown in Figure 4. The EI spectrum is obtained from the NIST database. Transform limited pulses with 35 fs time duration are used for fs-TOF and fs-LID experiments, with the peak power density of $4.0 \times 10^{15} \mathrm{~W} / \mathrm{cm}^{2}$ and $1.6 \times 10^{14} \mathrm{~W} / \mathrm{cm}^{2}$, respectively. The difference in power density is due to different focusing parameters, attenuation, and rep rates.

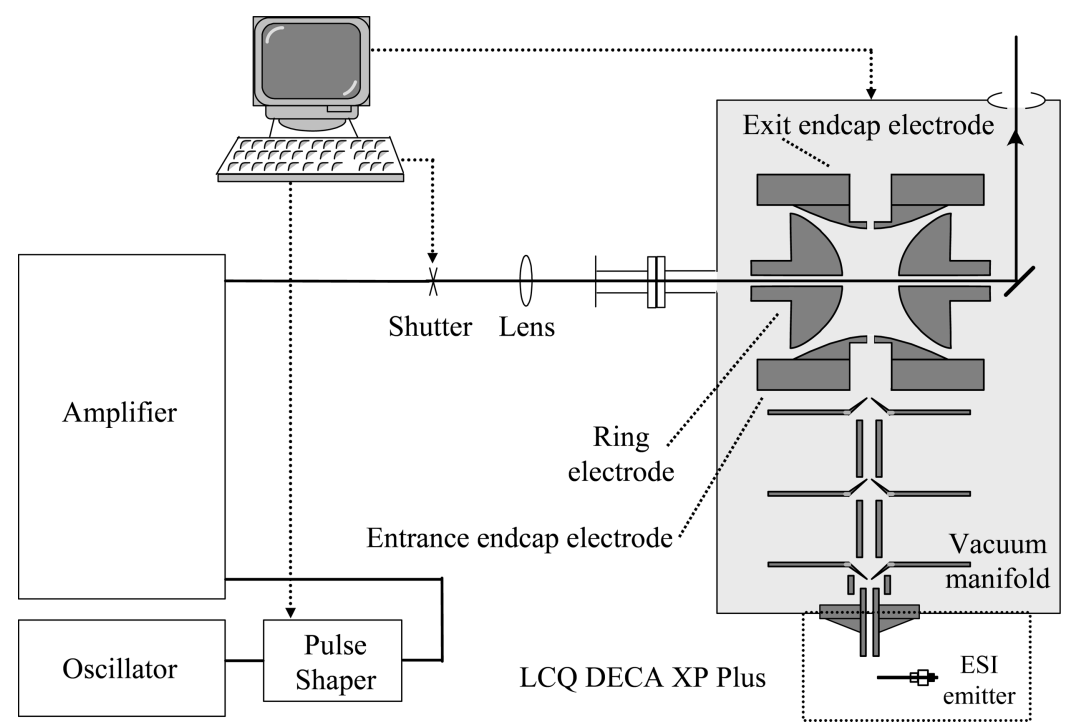

Figure 3. Schematic of the laser system and ion trap mass spectrometer used for fs-LID experiments. A viewport has been welded to the vacuum manifold and allows the laser beam to pass through a $5 \mathrm{~mm}$ hole drilled through the ring electrode of the ion trap, where it irradiates the ion cloud before being reflected out an additional window in the back of the instrument. 


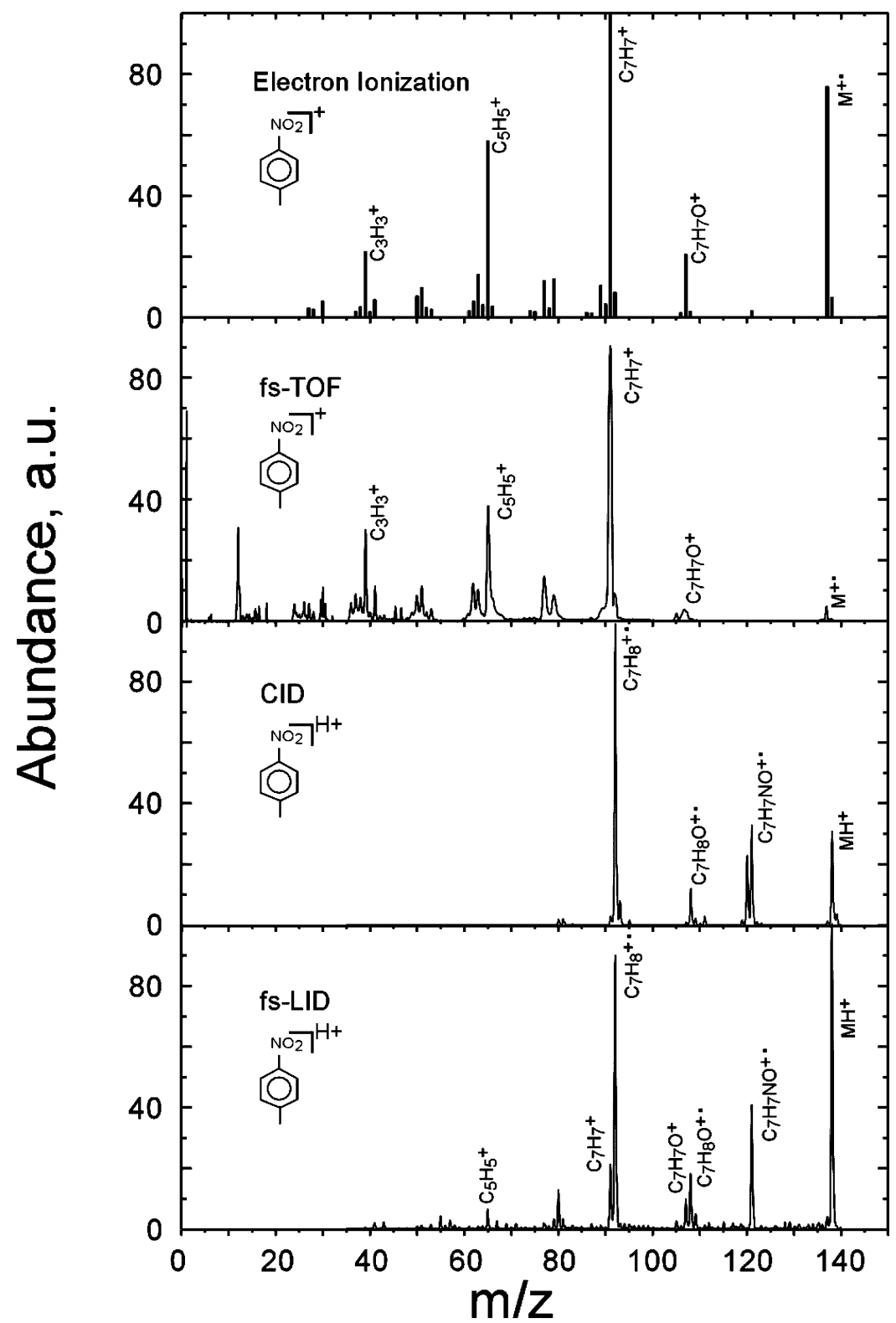

Figure 4. Mass spectra of $p \mathrm{NT}$ obtained by electron ionization, fs-TOF, CID, and fs-LID. Note that in the ion trap experiments (CID and fs-LID), the trapping potentials create a low mass cutoff of $35 \mathrm{~m} / \mathrm{z}$; any product ions generated below this point are ejected from the trap before the detection scan and therefore do not appear in the spectra.

The photochemistry of $p$ NT has been studied intensively in the past. ${ }^{19,20}$ In the fs-TOF experiments, $p$ NT molecules are fieldionized instantaneously. The molecular ions undergo isomerization in which $\mathrm{CH}_{3}-\phi-\mathrm{NO}_{2}$ transforms to $\mathrm{CH}_{3}-\phi-\mathrm{O}-\mathrm{NO}$. This mechanism is consistent with the observation of $\mathrm{CH}_{3}-\phi$ $\mathrm{O}^{+}$and the competition between the production of $\mathrm{NO}_{2}$ and $\mathrm{NO}$, since the dissociation could reach both products through a common transition state. ${ }^{19}$ In the presence of a strong field, the main channel following the isomerization is the elimination of $\mathrm{NO}_{2}$. The resulting $\mathrm{C}_{7} \mathrm{H}_{7}{ }^{+}$ions exist as tolyl and tropylium ions, which was confirmed by their absorption bands at 263 and 353 $\mathrm{nm}$ respectively. ${ }^{20}$ Sequential absorption of photons releases $\mathrm{C}_{2} \mathrm{H}_{2}$ fragments, giving rise to $\mathrm{C}_{5} \mathrm{H}_{5}{ }^{+}, \mathrm{C}_{3} \mathrm{H}_{3}{ }^{+}$, and $\mathrm{C}^{+}$ions.

The fragmentation pattern of $p \mathrm{NT}$ from electron ionization (Figure 4) is very similar to that obtained in fs-TOF with transform-limited pulses. However, fewer molecular ion and more small fragments $\left(\mathrm{C}^{+}, \mathrm{H}^{+}\right)$are observed in the fs-TOF spectrum. This is due to the absorption of additional photons by larger fragments, including molecular ions, resulting in further fragmentation.
The ion trap experiments on protonated $p$ NT reveal that the protonated molecule $\left(\mathrm{MH}^{+}\right)$follows a different fragmentation pathway than the ionized neutral $\left(\mathrm{M}^{+\bullet}\right)$. The fs-LID spectrum (Figure 4) shows a combination of even- and odd-electron product ions, corresponding primarily to losses involving heteroatoms from the nitro group. The peak at $121\left(\mathrm{C}_{7} \mathrm{H}_{7} \mathrm{NO}^{+\bullet}\right)$, resulting from the loss of $\mathrm{OH}$, is absent in the fs-TOF spectrum, but all other fs-LID product ions match up with fs-TOF product ions, with an occasional shift of $1 \mathrm{~m} / \mathrm{z}$ due to the retention of an extra proton. The tropylium ion is a minor product in fsLID, and therefore, the benzene ring fragment ions (such as $\mathrm{C}_{5} \mathrm{H}_{5}{ }^{+}$) are also in low abundance. Although smaller fragments may form upon activation with the femtosecond laser, the trapping voltages on the ring and end-cap electrodes impose a low mass cutoff of $35 \mathrm{~m} / \mathrm{z}$ in the ion trap; therefore, the smaller ions cannot be detected in this experiment.

Whereas CID is a commonly employed activation method for biomolecules, it does not lead to extensive fragmentation of small organics, such as $p$ NT. Nevertheless, the CID spectrum is included in Figure 4 to provide an additional comparison to 
a) fs-TOF

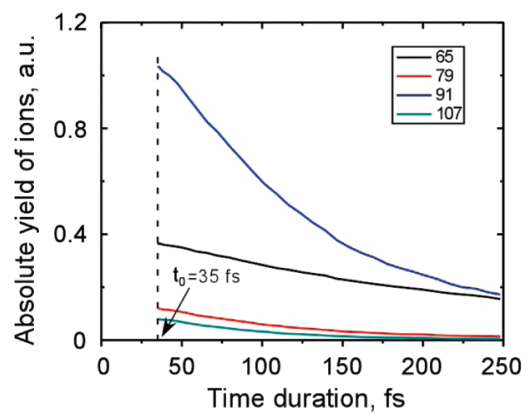

b) fs-LID
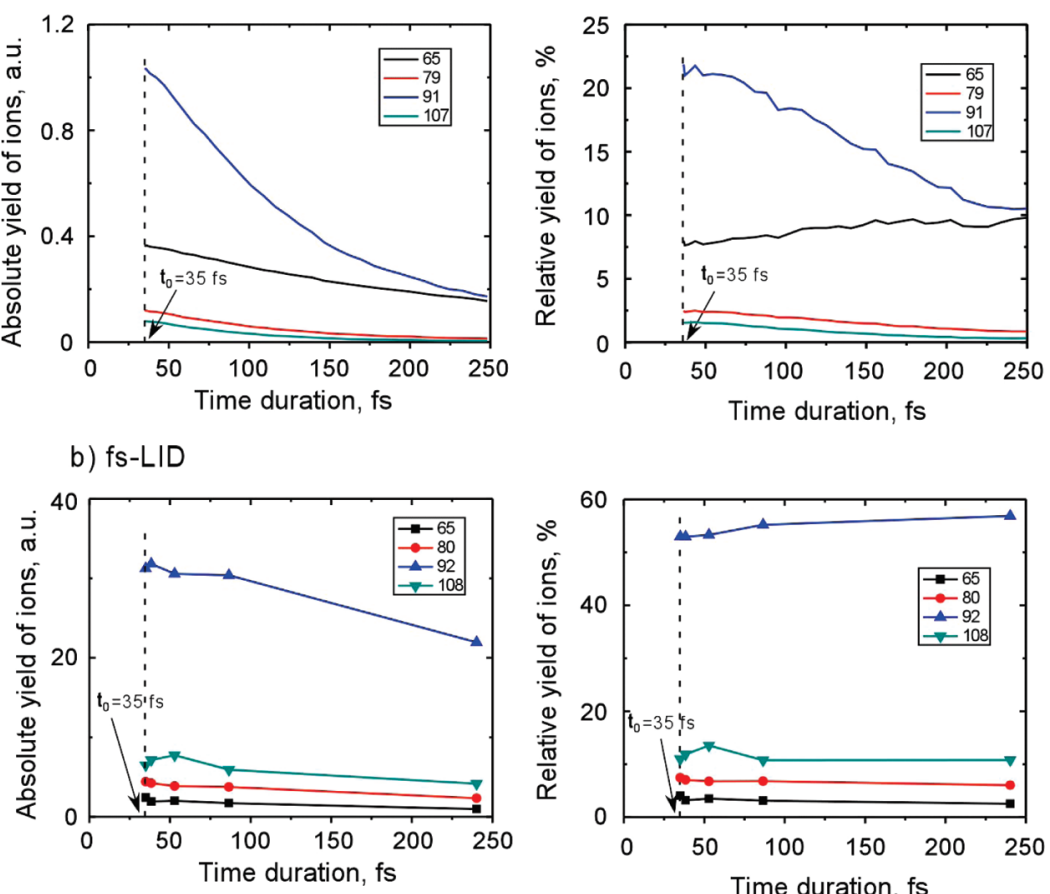

Figure 5. Chirp dependence of $p$ NT fragmentation obtained by fs-TOF (panel a) and fs-LID (panel b). Absolute and relative yields of several major product ions are plotted as a function of the time duration.

fs-LID. CID results in losses of $\mathrm{OH}, \mathrm{H}_{2} \mathrm{O}, \mathrm{NO}$, and $\mathrm{NO}_{2}$ from $p \mathrm{NT}$. We see no tropylium ion and, therefore, observe no benzene ring fragmentations. The only CID peak absent in the fs-LID spectrum corresponds to the water loss (peak 120), which comes from the protonated $\mathrm{NO}_{2}$ group grabbing an additional proton from the benzene ring and kicking out a water molecule, leaving $\mathrm{C}_{7} \mathrm{H}_{6} \mathrm{NO}^{+}$, which provides no additional structural information.

Mass spectra of $p \mathrm{NT}$ were measured while systematically changing the amount of linear chirp introduced into the pulses. A constant pulse energy was maintained so that larger linear chirp results in longer pulses with lower peak intensity. The absolute and relative yields of several major fragments are plotted as a function of pulse duration in Figure 5.

In fs-TOF experiments, absolute yields of all fragment ions decrease when the pulse duration increases. For longer pulses, the volume in which the power density is higher than the ionization threshold is smaller; thus, fewer molecules are ionized/activated and fragmented. On the other hand, the relative yields of larger fragments decrease and those of smaller fragments increase for longer pulses. The longer the pulses, the longer the time available for ions to absorb additional photons and fragment further.

Unlike the fs-TOF chirp dependence, the fs-LID experiments show that short, TL pulses are ideal for ion activation and dissociation in an ion trap. Longer pulses result in a loss of overall fs-LID signal, with all product ion intensities decreasing uniformly (as seen by the constant relative yields in Figure 5b). The difference between the TOF and ion trap data may be attributed to the dramatically different vacuum regimes utilized in each. Fragment ions are formed in the TOFMS at $10^{-5}$ Torr and can continue to dissociate into smaller and smaller pieces; however, helium gas maintains a pressure around 1 mTorr in the ion trap, so as product ions are formed, they undergo collisional cooling and are stored in the trap until the detection scan.
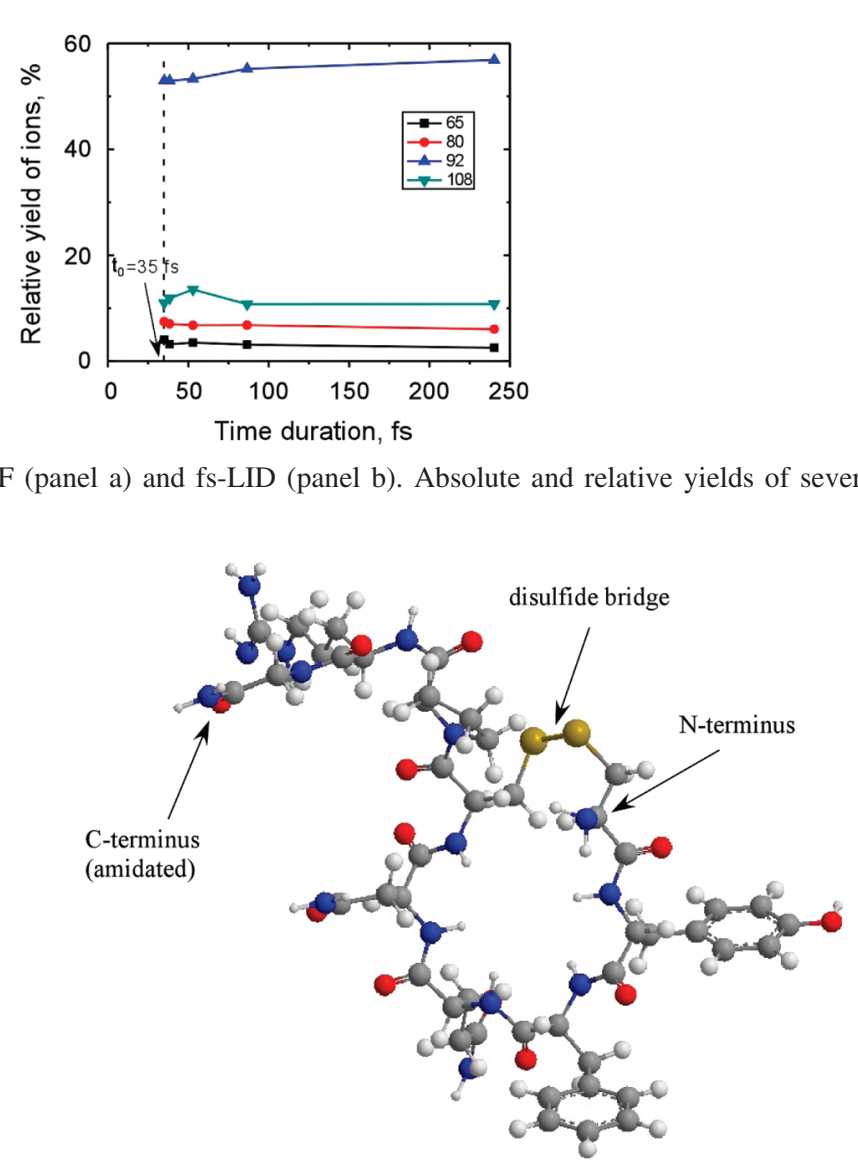

Figure 6. $\mathrm{Arg}^{8}$-vasopressin, $\mathrm{C}_{46} \mathrm{H}_{65} \mathrm{~N}_{15} \mathrm{O}_{12} \mathrm{~S}_{2}$, is a mammalian hormone and neurotransmitter. Its structure contains a disulfide bridge between cysteine residues in positions 1 and 6 . The single letter amino acid abbreviation for this molecule is CYFQNCPRG.

Proteomics: Results on Vasopressin. $\mathrm{Arg}^{8}$-vasopressin (sequence CYFQNCPRG-NH$H_{2}$ ) is one of more than 30 peptides that have been analyzed by fs-LID in the Dantus lab to date. The intramolecular disulfide bridge between the cysteine residues at positions 1 and 6 is of particular interest because of the rigidity it lends to the peptide structure (Figure 6). The covalent $\mathrm{S}-\mathrm{S}$ bond is strong and typically will not break during activation by CID, making disulfide mapping in larger peptides and proteins difficult. As the CID spectrum (Figure 7A) of the singly protonated precursor demonstrates, this is problematic because any backbone cleavages induced between the cysteine residues will not actually fragment the molecule: the two pieces will remain linked by the disulfide bridge and be detected at the same $m / z$ as the precursor ion population. As a result, the CID spectrum contains very little sequence information, with 


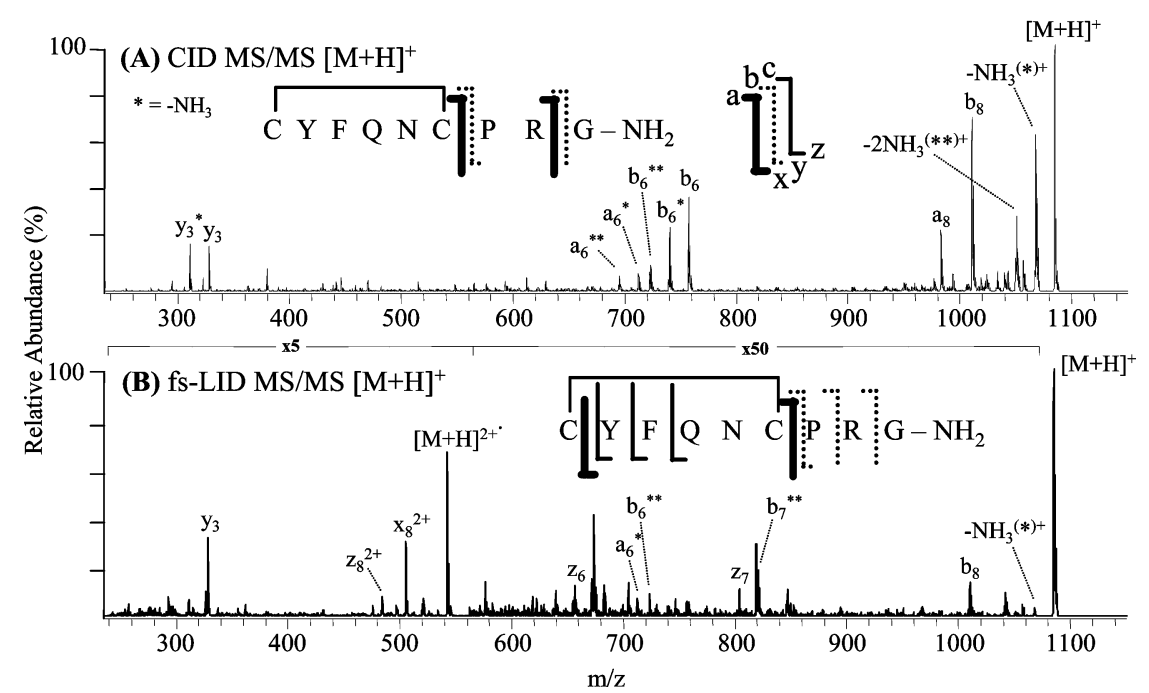

Figure 7. CID (A) and fs-LID (B) spectra of Arg8-vasopressin. Standard peptide fragmentation nomenclature is utilized, with a, b, c, $x, y$, and z ions referring to different cleavages along the peptide backbone, while the subscripts indicate how many amino acid residues are retained in each product ion.

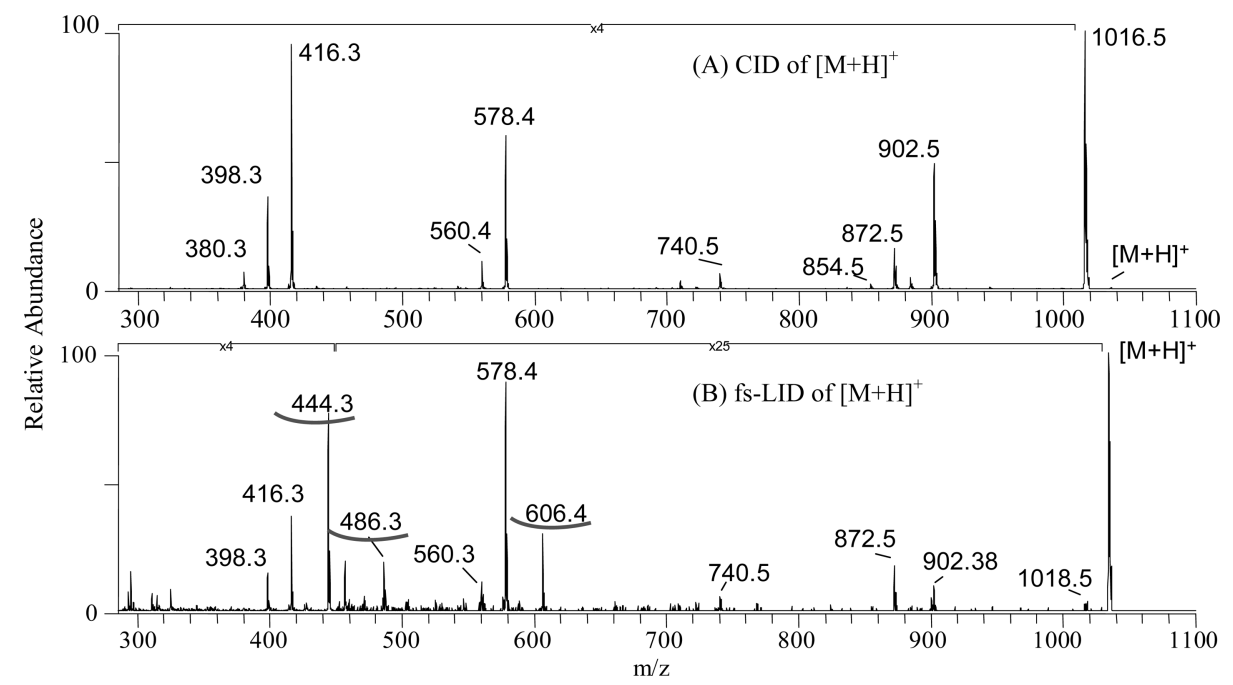

Figure 8. MS/MS spectra of tomatine using CID (A) and fs-LID (B). Labeled peaks are those of carbohydrate loss, ring cleavage, and water loss. The three cross-ring fragment peaks unique to fs-LID are underlined.

the few product ions corresponding to cleavages near the C-terminal end of the peptide.

Alternatively, fs-LID greatly increases the information content of the MS/MS spectrum (Figure 7B). The dominant product ion is $[\mathrm{M}+\mathrm{H}]^{2+\bullet}$, formed through the photoionization of the precursor ion. This radical is a likely intermediate for many of the other product ions. The observation of the $\mathrm{x}$ - and $\mathrm{z}$-type ions cleavages denoted at the $\mathrm{N}$-terminal end of the peptide indicates that the disulfide bridge has been broken in addition to cleavages along the peptide backbone. This supports the finding that fs-LID fragments analytes in a nonstatistical manner. If the laser irradiation were simply causing thermal heating of the trapped precursor ions, we would expect to break only the weakest bonds, which are the peptide bonds along the vasopressin backbone. However, we see several product ions in the fs-LID spectrum in which the very strong $S-S$ bond has been broken, yet many of the amino acids remain linked together $\left(\mathrm{x}_{8}, \mathrm{z}_{6}, \mathrm{z}_{7}\right.$, and $\mathrm{z}_{8}$ ions). The ability to cleave strong bonds while leaving weaker bonds intact makes fs-LID a promising tool for PTM analysis.

Metabolomics: Results on Tomatine. Glycosylation, a PTM mentioned earlier, is common not only for proteins, but also for lipids and other organic molecules. Carbohydrate moieties can contribute important characteristics to a molecule, but can be difficult to study due to the many possible isomers and branching patterns. $\propto$-Tomatine (chemical formula $\mathrm{C}_{50} \mathrm{H}_{83} \mathrm{NO}_{21}$ ), a steroidal glyco-alkaloid, is a toxin found in tomato plants. Tomatine has been found to inhibit the growth of cancer cells, ${ }^{21,22}$ yet the same molecule without the carbohydrate chain (tomatidine) has little to no effect on cancer inhibition. ${ }^{22}$

Traditional means of carbohydrate MS involve covalent modification, such as methylation, acetylation, or trimethylsilylation, to obtain information-rich mass spectra. As seen in Figure 8, CID of unmodified tomatine offers little information as to which glyco-constitutional isomers may be present in the molecule. The spectrum obtained by CID shows mainly loss of whole sugar groups $(416.3,578.4,740.5,872.5,902.5)$ due to cleavage of weak glycosidic bonds, as well as almost compete loss of water from the parent ion (1016.5), and loss of water molecules throughout the carbohydrate $(560.4,854.5,884.5)$ and steroid portions of the molecule $(380.3,398.3)$. Glycosidic bond cleavage yields only the chemical formula of the losses, and water loss yields no structural information. 

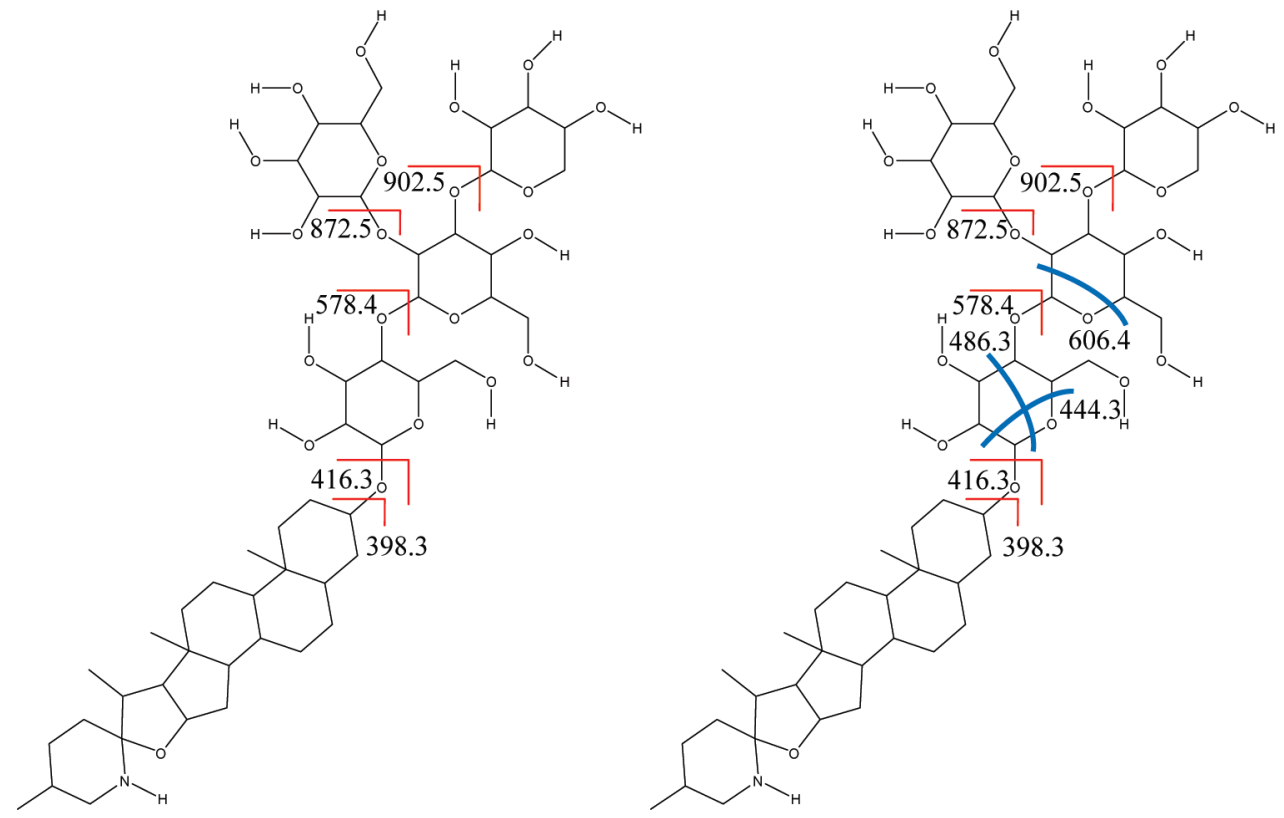

Figure 9. Comparison of CID and fs-LID fragmentation of $\alpha$-tomatine. Cleavages found in both CID and fs-LID are marked with right angles; those unique to fs-LID are curved.

Fs-LID displays cross-ring cleavages of the carbohydrate monomers $(444.3,486.3,606.4)$; minimal loss of water from the parent ion, independent of laser power; and suppressed water loss from the steroid backbone (Figure 8). In addition, these unique fs-LID products are formed without sacrificing useful information from the CID spectrum, as shown by a comparison of their bond cleavages in Figure 9. These complementary fragments may be useful in determining carbohydrate branching patterns and differentiating between conformational isomers, allowing elucidation of the monomers and identification of the entire carbohydrate in various glycometabolites and carbohydratebased PTMs.

The additional information provided by fs-LID comes without the need for wet chemistry, such as covalent modifications, or chromophores, which are necessary for other laser-based experiments.

\section{Conclusion}

The work presented here bridges three different fields, each one with fundamentally different perspectives. Starting with femtochemistry, the goal is to probe ultrafast molecular reaction dynamics. The field of laser control of chemical reactions is concerned with directing the molecular dynamics to close undesired reaction pathways while opening or optimizing a reaction pathway that leads to a desired product. Finally, the field of biological mass spectrometry struggles to find new methods for inducing ion fragmentation. The common thread among these three fields is the use of ultrafast, $\tau<40 \mathrm{fs}$, pulses for depositing energy into the molecule on a time scale that is faster than molecular vibrations.

In all cases, it is critical to have control over the temporal shape of the laser pulse to maximize signal and provide reproducible and reliable mass spectra. The key result we want to highlight here is that using an ultrashort laser for activation provides mass spectra with greater information content than conventional activation methods. This is useful for identification and sequencing in the case of peptides. Using pulses of different shapes is shedding light on the intramolecular behavior of small ions and also provides additional information for identification.
Ultrashort laser activation in all cases demonstrates nonstatistical pathways of dissociation for all ions studied. This can open many doors to understanding and improvements in the field of mass spectrometry, as well as in fundamental chemical physics and the physical chemistry of macromolecules. We think that using ultrafast optical activation as an additional tool will lead to progress in the fields of proteomics and metabolomics, especially in post translational modification analysis of proteins and medical diagnostics.

Acknowledgment. Support for this work comes from Grant 156 of the 21 st Century Jobs Trust Fund of the SEIC Board from the State of Michigan and from Grants CHE-0647901 from the National Science Foundation. The authors thank Professor Gavin Reid and Professor Dan Jones for their valuable discussions on protein and metabolomic analysis.

\section{References and Notes}

(1) Zewail, A. J. Phys. Chem. A 2000, 104, 5660.

(2) Kalcic, C. L.; Gunaratne, T. C.; Jones, A. D.; Dantus, M.; Reid, G. E. J. Am. Chem. Soc. 2009, 131, 940.

(3) Oh, J. Y.; Moon, J. H.; Kim, M. S. Rapid Commun. Mass Spectrom. 2004, 18, 2706.

(4) Reilly, J. P. Mass Spectrom. Rev. 2009, 28, 425.

(5) Gregoire, G.; Kang, H.; Dedonder-Lardeux, C.; Jouvet, C.; Desfrancois, C.; Onidas, D.; Lepere, V.; Fayeton, J. A. Phys. Chem. Chem. Phys. 2006, 8, 122.

(6) Kang, H.; Jouvet, C.; Dedonder-Lardeux, C.; Martrenchard, S.; Gregoire, G.; Desfrancois, C.; Schermann, J. P.; Barat, M.; Fayeton, J. A. Phys. Chem. Chem. Phys. 2005, 7, 394.

(7) Guyon, L.; Tabarin, T.; Thuillier, B.; Antoine, R.; Broyer, M.; Boutou, V.; Wolf, J. P.; Dugourd, P. J. Chem. Phys. 2008, 128.

(8) Zubarev, R. A.; Kelleher, N. L.; McLafferty, F. W. J. Am. Chem. Soc. $1998,120,3265$.

(9) Syka, J. E. P.; Coon, J. J.; Schroeder, M. J.; Shabanowitz, J.; Hunt, D. F. Proc. Natl. Acad. Sci. 2004, 101, 9528.

(10) Budnik, B. A.; Zubarev, R. A. Chem. Phys. Lett. 2000, 316, 19.

(11) Lezius, M.; Blanchet, V.; Ivanov, M. Y.; Stolow, A. J. Chem. Phys. 2002, 117, 1575 .

(12) Hankin, S. M.; Villeneuve, D. M.; Corkum, P. B.; Rayner, D. M. Phys. Rev. Lett. 2000, 84, 5082.

(13) Hankin, S. M.; Villeneuve, D. M.; Corkum, P. B.; Rayner, D. M. Phys. Rev. A 2001, 64.

(14) Assion, A.; Baumart, T.; Bergt, M.; Brixner, T.; Kiefer, B.; Sayfried, V.; Strehle, M.; Gerber, G. Science 1998, 282, 919. 
(15) Lozovoy, V. V.; Zhu, X.; Gunaratne, T. C.; Harris, D. A.; Shane, J. C.; Dantus, M. J. Phys. Chem. A 2008, 112, 3789.

(16) Laarmann, T.; Shchatsinin, I.; Singh, P.; Zhavoronkov, N.; Gerhards, M.; Schulz, C. P.; Hertel, I. V. J. Chem. Phys. 2007, 127, 201101.

(17) Biemann, K. Methods Enzymol. 1990, 193, 886.

(18) Coello, Y.; Lozovoy, V. V.; Gunaratne, T. C.; Xu, B. W.; Borukhovich, I.; Tseng, C. H.; Weinacht, T.; Dantus, M. J. Opt. Soc. Am. B 2008, 25, A140.

(19) Galloway, D. B.; Bartz, J. A.; Huey, L. G.; Crim, F. F. J. Chem. Phys. 1993, 98, 2107.
(20) Lablanquie, P.; Ohashi, K.; Nishi, N. J. Chem. Phys. 1993, 98 , 399.

(21) Friedman, M.; McQuistan, T.; Hendricks, J. D.; Pereira, C.; Bailey, G. S. Mol. Nutr. Food Res. 2007, 51, 1485.

(22) Friedman, M.; Levin, C. E.; Lee, S. U.; Kim, H. J.; Lee, I. S.; Byun, J. O.; Kozukue, N. J. Agric. Food Chem. 2009, 57, 5727.

JP1000732 